Published in final edited form as:

Eur Urol. 2015 January ; 67(1): 165-170. doi:10.1016/j.eururo.2014.01.009.

\title{
Trends in the Use of Perioperative Chemotherapy for Localized and Locally Advanced Muscle-invasive Bladder Cancer: A Sign of Changing Tides
}

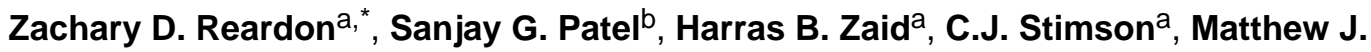 \\ Resnick $^{a, c, d}$, Kirk A. Keegan ${ }^{e}$, Daniel A. Barocas ${ }^{a, c}$, Sam S. Chang ${ }^{a}$, and Michael S. \\ Cookson $^{\dagger}$ \\ aDepartment of Urologic Surgery, Vanderbilt University Medical Center, Nashville, TN, USA \\ bSection of Urology, University of Chicago Medical Center, Chicago, IL, USA \\ ${ }^{\circ}$ Center for Surgical Quality and Outcomes Research, Vanderbilt University Medical Center, \\ Nashville, TN, USA \\ dThe VA Tennessee Valley Health Care System, Nashville, TN, USA \\ eDivision of Urology, San Antonio Military Medical Center, San Antonio, TX, USA \\ fDepartment of Urology, University of Oklahoma College of Medicine, Norman, OK, USA
}

\section{Abstract}

\begin{abstract}
Background-Despite the documented survival benefit conferred by neoadjuvant (NAC) and adjuvant chemotherapy (AC), there has been a slow adoption of guideline recommendations for the use of perioperative chemotherapy (POC) in patients with muscle-invasive bladder cancer (MIBC).
\end{abstract}

\footnotetext{
(C) 2014 Published by Elsevier B.V. on behalf of European Association of Urology.

*Corresponding author. Vanderbilt University Medical Center, 1161 21st Avenue South, Nashville, TN 37232-2765. Tel. +1 615322 2101; Fax: +1 615322 5869. zachary.d.reardon@ vanderbilt.edu (Z.D. Reardon).

Author contributions: Zachary D. Reardon had full access to all the data in the study and takes responsibility for the integrity of the data and the accuracy of the data analysis.

Study concept and design: Reardon, Zaid, Stimson, Patel, Barocas, Keegan, Resnick, Chang, Cookson.

Acquisition of data: Reardon, Zaid, Stimson, Patel.

Analysis and interpretation of data: Reardon, Zaid, Stimson, Patel, Barocas, Keegan, Resnick, Chang, Cookson.

Drafting of the manuscript: Reardon, Zaid, Patel.

Critical revision of the manuscript for important intellectual content: Reardon, Zaid, Stimson, Patel, Barocas, Keegan, Resnick,

Chang, Cookson.

Statistical analysis: Reardon, Zaid, Stimson, Patel, Barocas, Resnick.

Obtaining funding: Resnick and Keegan.

Administrative, technical, or material support: Zaid, Resnick, Barocas, Chang, Cookson.

Supervision: Barocas, Resnick, Chang, Cookson.

Other (specify): None.

Financial disclosures: Zachary D. Reardon certifies that all conflicts of interest, including specific financial interests and relationships and affiliations relevant to the subject matter or materials discussed in the manuscript (eg, employment/affiliation, grants or funding, consultancies, honoraria, stock ownership or options, expert testimony, royalties, or patents filed, received, or pending), are the following: Zachary D. Resnick is a consultant/advisor for Photocure and Dendron. Daniel A. Barocas is a consultant/advisor for GE Healthcare, Janssen, and Dendreon. Chang is a consultant/advisor for Photocure and EndoEthicon.
} 
Objective-To evaluate temporal trends in POC utilization and identify factors influencing POC delivery in a representative cohort of patients with MIBC.

Design, setting, and participants-Retrospective cohort study identifying factors associated with receipt of POC and evaluating temporal changes in NAC and AC utilization. We included patients from the National Cancer Data Base (NCDB) with no prior malignancy who ultimately underwent radical cystectomy for $\geq \mathrm{T} 2 / \mathrm{cN} 0 / \mathrm{cM} 0$ MIBC between 2006 and 2010.

Outcome measurements and statistical analysis-Relationships between demographic and hospital factors and the likelihood of receiving POC were evaluated using Pearson chi-square and Wilcoxon rank-sum tests, and multivariable logistic regression. Temporal changes in NAC and $\mathrm{AC}$ use were detected using a linear test of trend.

Results and limitations-A total of 5692 patients met our inclusion criteria. POC use increased from $29.5 \%$ in 2006 to $39.8 \%$ in 2010 ( $p<0.001)$. NAC use increased from $10.1 \%$ in 2006 to $20.8 \%$ in 2010 ( $p=0.005$ ); AC remained stable between $18.1 \%$ and $21.3 \%$ ( $p=0.68$ ). Multivariable modeling revealed advanced age, increasing comorbidity, lack of insurance, increased travel distance, geographic location outside the northeastern United States, and lower income as negatively associated with POC receipt (all $p<0.05$ ). Limitations include retrospective design and potential sampling bias, excluding patients treated at non-NCDB facilities.

Conclusions-POC use for MIBC increased from 2006 to 2010, with this increase disproportionately due to rising NAC utilization. Nonetheless, there is persistent variation in the likelihood of receiving POC secondary to nonclinical factors.

Patient summary-When retrospectively analyzing a representative cohort of patients undergoing radical cystectomy for muscle-invasive bladder cancer between 2006 and 2010, we noted that preoperative chemotherapy rates increased steadily while use of chemotherapy after surgery remained stable. Factors related to access to care significantly influenced receipt of chemotherapy.

\section{Keywords}

Bladder cancer; Neoadjuvant therapy; Adjuvant therapy; Drug therapy; Review

\section{Introduction}

In the United States and the European Union, bladder cancer (BCa) accounts for approximately 180000 new cancer diagnoses and >50 000 deaths annually [1]. At the time of diagnosis, 25-30\% of bladder tumors are found to be muscle invasive [1,2]. Radical cystectomy (RC) remains the preferred treatment for patients with muscle-invasive bladder cancer (MIBC). The use of perioperative chemotherapy (POC), defined as either neoadjuvant (NAC) or adjuvant chemotherapy (AC), has been associated with improved survival in select patients undergoing RC [3,4]. As such, the use of POC has been included in contemporary guidelines on the management of MIBC [5,6].

Prior analysis suggests that application of POC for MIBC has been low and inconsistent [7]. Level 1 evidence from the SWOG-8710 trial and subsequent meta-analyses have demonstrated improved survival and pathologic down-staging with the use of chemotherapy 
prior to RC [3,8,9]. Despite this evidence, adoption of NAC for MIBC has been slow. A review of Medicare beneficiaries treated between 1992 and 2002 demonstrated that only $1.4 \%$ and $11 \%$ of cT2 and cT4 patients received NAC, respectively [10]. Evidence for the use of $\mathrm{AC}$ is variable, with some studies demonstrating survival advantage associated with postoperative chemotherapy and others failing to demonstrate such an advantage. A metaanalysis of six trials that randomized patients with cT2-cT4 BCa to adjuvant cisplatin-based chemotherapy or observation after local definitive therapy suggested a $25 \%$ relative reduction in the risk of death for patients who received chemotherapy [4]. This result was confirmed in a more recent meta-analysis of nine randomized control trials that revealed a $23 \%$ improvement in overall survival and $44 \%$ improvement in disease-free survival with the use of chemotherapy following RC for MIBC [11].

Currently there exist few data evaluating patterns of care with respect to systemic therapy in patients treated after the 2003 publication of the landmark SWOG- 8710 trial. We sought to assess whether there has been a shift in overall POC use over time as well as to determine the proportional change in NAC and AC utilization using the National Cancer Data Base (NCDB) registry, with data from 2006 to 2010 . Additionally, we sought to identify the influence of nonclinical patient and hospital characteristics on the receipt of POC for MIBC.

\section{Material and methods}

The NCDB is a hospital-based cancer registry sponsored by a joint program between the Commission on Cancer $(\mathrm{CoC})$ of the American Cancer Society and the American College of Surgeons. This database captures $>70 \%$ of all new cancer diagnoses in the United States and draws from $>1400$ facilities. Data collected include demographic and clinical patient characteristics, cancer staging, and tumor histology [12]. Institutional review board approval was not required for this study given that no patient, physician, or hospital identifiers were examined.

We identified 542053 patients diagnosed with BCa in the NCDB registry between 1998 and 2010. Using morphological codes from the International Classification of Diseases for Oncology, 3rd edition, we limited our cohort to patients with histologically proven urothelial carcinoma who received treatment at reporting institutions between 2006 and 2010 ( $n=202$ 422). The time period 2006-2010 was chosen to provide an updated analysis because previous studies commented on chemotherapy utilization prior to this period $[7,10]$. Our cohort was further restricted to patients with clinical $\geq T 2$ tumors in the absence of nodal $(\mathrm{cN} 0)$ or distant metastatic disease $(\mathrm{cM} 0)(\succeq \mathrm{T} 2 / \mathrm{cN} 0 / \mathrm{cM} 0)$ and no prior history of malignancy $(n=9811)$. Finally, we identified those patients who underwent RC for definitive management of their MIBC $(n=5692)$. Patients undergoing RC for <cT2 disease were not included because no clear indication for NAC exists for these patients.

Although specific chemotherapeutic agents and number of treatments cycles are not detailed in the NCDB registry, the timing of systemic chemotherapy is coded with relation to definitive surgical therapy, allowing for the accurate identification of NAC and AC. The overall rate of POC utilization included patients who received chemotherapy in the neoadjuvant and adjuvant settings. Temporal trends in the use of POC were calculated as the 
proportion of patients receiving NAC, AC, or no systemic therapy annually. A linear test of trend was performed to detect differences in the rate of chemotherapy delivery over time.

To identify factors associated with the receipt of POC, we compared patients who received NAC plus RC or RC plus AC with those who received only RC. We evaluated the relationship between demographic factors including age, gender, race, insurance status, income, and rural location and the receipt of POC. Race was categorized as non-Hispanic white, African American, or other/unknown; patient insurance status as uninsured, private, Medicaid, Medicare, Veterans Affairs/Military, and other/unknown; and income as $<\$ 30$ 000, \$30 000-34 999, \$35 000-45 999, and $\$ \$ 46000$ annually. Rural-urban continuum codes were classified by matching the state and county Federal Information Processing Standard code where a patient received treatment against files published by the US Department of Agriculture Economic Research Service.

Patient comorbidity was estimated using the Charlson Comorbidity Index (CCI), and was calculated based on the reporting of up to six preexisting comorbidities as recorded on the hospital discharge summary, excluding the patient's cancer diagnosis and categorized as 0 (no significant reported comorbidity), 1 , or $>1$.

We also considered the relationships between receipt of POC and geographic location, patient distance from treatment facility, and type of treating facility. Location was divided into regions based on the state in which the reporting institution is based: Northeast, Southeast, Midwest, and West. Distance from treatment facility was reported in miles between patient's zip code and the treatment facility. The type of treating hospital is designated by the $\mathrm{CoC}$ and includes community centers (between 100 and 500 cancer patients treated annually), comprehensive community centers ( $>500$ cancer patients treated annually), and academic centers ( $>500$ cancer patients treated annually, with an associated graduate medical education program).

Bivariate relationships were elaborated using the Pearson chi-square test and the Wilcoxon rank-sum test for categorical and continuous variables, respectively. We performed multivariable logistic regression analysis with receipt of POC as the response variable and year of diagnosis, age, CCI, race, insurance status, distance from treatment facility, geographic location, type of treating hospital, income, and rurality index as covariates. Similar nonclinical features were previously identified as potential influences on treatment patterns for MIBC [10]. All statistical analyses were performed using Stata software v.11.1 (StataCorp, College Station, TX, USA). All $p$ values are reported as two sided, and $p$ values $<0.05$ were considered statistically significant.

\section{Results}

From the parent cohort of 542053 patients with BCa in the NCDB, 5692 met our inclusion criteria. The median age in our cohort was $65 \mathrm{yr}$ (interquartile range [IQR]: 14) and $69 \mathrm{yr}$ (IQR: 14) for those patients who did and did not receive POC, respectively.

In our cohort, 2056 of 5692 (36.1\%) received POC and 3636 of 5692 (63.9\%) did not receive chemotherapy. The mean time from diagnosis to RC was 3.3. mo (standard deviation 
[SD]: 1.6) for those who did not receive NAC compared with 3.8 mo (SD: 2.1) for those patients who did receive NAC ( $p<0.01$ ). POC use increased from $29.5 \%$ in 2006 to $39.8 \%$ in 2010 ( $p<0.001)$. When stratified by timing of delivery, NAC use steadily rose between 2006 and $2010(10.1-20.9 \% ; p=0.005)$; AC remained relatively stable during that same time period (range: $18.1-21.33 \% ; p=0.68$ ) (Fig. 1).

Table 1 shows the demographic patient features by treatment type. Distribution of gender and race was similar between patients who did and did not receive chemotherapy ( $p=0.59$ and $p=0.81$, respectively). Patients receiving NAC and AC were noted to be significantly younger, more likely to have private insurance, receive treatment at an academic hospital, and had higher annual income compared with patients who underwent $\mathrm{RC}$ alone (all $p$ values $<0.05$ ).

We performed multivariable logistic regression analysis to identify factors associated with POC (Table 2). Later year of diagnosis was associated with increased likelihood of POC administration (odds ratio [OR]: $1.11 ; p<0.01$ ). Compared with patients $<50 \mathrm{yr}$ of age, the odds of receiving POC decreased significantly for patients $>60 \mathrm{yr}$. Patients with a CCI of 1 and CCI $>1$ were less likely to receive POC (ORs: 0.86 and 0.64 , respectively; $p<0.05$ for each). Uninsured patients were less likely to receive POC than patients with private insurance or managed care (OR: $0.64 ; p<0.01)$. Patients treated in the US South, Midwest, and West were significantly less likely to receive POC than patients treated in the Northeast (ORs: $0.70,0.79$, and 0.64, respectively; $p<0.01$ for all). Patient residence $₹ 60$ miles from the treatment facility was also negatively associated with receipt of POC (OR: $0.64 ; p<$ 0.05). Annual income $\$ 35000$ was associated with increased likelihood to receive POC compared with patients with lower incomes. Patient gender, race, rural location, and hospital type were not independently associated with the administration of POC.

\section{Discussion}

Our study identifies an important shift in systemic treatment patterns for MIBC over the last decade. Overall chemotherapy administration increased from 29.5\% to 39.8\% between 2006 and $2010(p<0.001)$, representing a relative increase of $35.0 \%$. When stratified by timing of chemotherapy delivery, it appears this increase is driven by rising NAC administration.

In comparison with similar studies in patients treated prior to 2003, there appears to be a clear change in practice patterns. A previous NCDB analysis of POC use in patients with stage III BCa from 1999 to 2003 revealed that $11.6 \%$ of patients received POC, with $1.2 \%$ and $10.4 \%$ receiving NAC and $\mathrm{AC}$, respectively. An increase in POC from 11.3\% to $16.8 \%$ was noted over this same time period [7]. A follow-up NCDB evaluation revealed that overall NAC use increased from 6\% to $13 \%$ between 2003 and 2007, with no change in AC use [13]. Our analysis of the same data source demonstrates that, although utilization of POC has not been uniformly applied, there appears to be increasing adoption of evidencebased clinical practice. This change in practice patterns with a time lag following guideline publication is consistent with the theory of diffusion of innovation, a process by which new technology or practices spread through social networks of physicians and patients over time [14]. 
Several hypotheses exist regarding the relatively slow adoption of POC use in MIBC.

Certainly, the significant toxicities noted in the standard methotrexate, vinblastine, doxorubicin, cisplatin regimen used in the SWOG-8710 trial tempered enthusiasm for the ubiquitous use of NAC. Administrative claims data have shown considerable declines in methotrexate and vinblastine administration coupled with increased paclitaxel, gemcitabine, and carboplatin use since the mid-1990s [10]. This is most certainly due to the more favorable toxicity profiles associated with contemporary treatment regimens [10].

$\mathrm{BCa}$ is typically a disease of older adults as demonstrated by the mean age of our cohort (66.6 yr), a number corroborated by prior NCDB and Surveillance Epidemiology and End Results (SEER) analyses $[2,10]$. In our evaluation, increasing age was negatively associated with receipt of POC. A retrospective evaluation of patients with inoperable locally advanced, metastatic, or recurrent BCa between 1995 and 2003 suggested that patients $\geq 70$ yr of age tolerate standard platinum-based chemotherapy regimens and receive similar oncologic benefits compared with younger patients [15]. Further data have demonstrated a survival advantage for octogenarians managed with aggressive surgical intervention (RC), suggesting that patients of advanced age who can tolerate aggressive therapy may derive benefit [16]. Our analysis implies that practicing clinicians may be reluctant to administer POC to older patients because advancing age beyond $59 \mathrm{yr}$ was independently associated with decreased receipt of POC.

Despite movement toward chemotherapy regimens that are better tolerated, poor performance status and impaired renal function remain contraindications for platinum-based chemotherapy [17]. In our analysis, patient comorbidity as measured by the CCI was a predictor of lower POC delivery. Several measures of overall health including renal function, cardiovascular disease, pulmonary disease, and hepatic dysfunction are incorporated into the CCI [18]. Although the NCDB does not directly report performance status, CCI may serve as an adequate surrogate measure [19].

Previous publications have also noted several nonclinical barriers to the receipt of POC. A recently published SEER analysis evaluating BCa patients treated between 1992 and 2002 found that geographic region in the United States significantly influenced receipt of systemic chemotherapy [10]. An NCDB analysis published in 2013 revealed minority race, lower income, lack of insurance, and treatment at a low-volume hospital center as predictors of less aggressive therapy in patients with MIBC [20]. Our study confirms several nonclinical patient features significantly associated with receipt of POC. We noted that lack of insurance, residence outside of the northeastern United States, and increasing patient distance from the treatment facility were negatively associated with POC use. Furthermore, increasing annual income $>\$ 35,000$ predicted a higher likelihood of $\mathrm{POC}$ receipt.

Prior analyses demonstrate that between $30 \%$ and $50 \%$ of patients with MIBC are ineligible for standard chemotherapy due to impaired renal function and performance status [21-23]. By 2010, 39.8\% of our cohort received POC. Although a rising trend in guideline adherence is reassuring, the optimal rate of POC administration for MIBC is not known. In an era where physicians are subject to performance measures contingent on both quality-of-care metrics and patient outcomes, reconciliation of guideline adherence, patient selection, and 
execution of appropriate therapy in the setting of MIBC will become increasingly complex. It is clear that shared decision making between individual patients and physicians as well as a risk-stratified approach to the administration of POC will optimize outcomes.

Several limitations exist in our study. First, sampling bias may be present because the NCDB records data only from CoC-approved facilities. Our findings may not be generalizable to patients outside of CoC-approved hospitals. Review of data from several disease processes have revealed that patient and disease treatment characteristics recorded in the NCDB are largely similar to those reported in SEER, which is a nationally representative sample [24]. Additionally, as a large administrative database the NCDB lacks granular data points such as type of chemotherapy or number of cycles administered, renal function, and performance status that may lead to some confounding effect.

\section{Conclusions}

We demonstrate a significant increase in overall use of POC secondary to an essential doubling in NAC use among patients undergoing RC for nonmetastatic MIBC between 2006 and 2010. These data indicate a robust diffusion of level 1 data. Despite the apparent adoption of evidence-based practice, several barriers to high-quality BCa treatment exist for traditionally underserved populations such as the elderly, the uninsured, and low-income patients. Development of appropriate tools to prospectively stratify patient benefit from POC for MIBC will allow for well-informed patient-doctor decision making and optimize outcomes.

\section{Acknowledgments}

We would like to thank the Commission on Cancer for allowing us to access this database as well as Greer Gay for her database guidance.

Funding/Support and role of the sponsor: Zachary D. Resnick was supported by the Veterans Affairs National Quality Scholars Program with the use of facilities at the Veterans Health Administration Tennessee Valley Healthcare System and in part by a grant from the Urology Care Foundation Research Scholars Program and the AUA Southeastern Section Research Scholar Fund. Kirk A. Keegan was also supported in part by the National Institutes of Health, K-12 Paul Calabresi Career Development Award for Clinical Oncology. The funding organizations helped design and conduct the study.

\section{References}

1. Burger M, Catto JW, Dalbagni G, et al. Epidemiology and risk factors of urothelial bladder cancer. Eur Urol. 2013; 63:234-41. [PubMed: 22877502]

2. Howlader, N.; Noone, AM.; Krapcho, M., et al. SEER cancer statistics review, 1975-2009 (vintage 2009 populations). Bethesda, MD: National Cancer Institite; 2009.

3. Grossman HB, Natale RB, Tangen CM, et al. Neoadjuvant chemotherapy plus cystectomy compared with cystectomy alone for locally advanced bladder cancer. N Engl J Med. 2003; 349:859-66. [PubMed: 12944571]

4. Vale CL. Adjuvant chemotherapy in invasive bladder cancer: a systematic review and meta-analysis of individual patient data: Advanced Bladder Cancer (ABC) Meta-analysis Collaboration. Eur Urol. 2005; 48:189-99. discussion 199-201. [PubMed: 15939530]

5. NCCN clinical practice guidelines in oncology: bladder cancer. Fort Washington, PA: National Comprehensive Cancer Network; 2013. 
6. Stenzl A, Cowan NC, De Santis M, et al. Treatment of muscle-invasive and metastatic bladder cancer: update of the EAU guidelines [in Spanish]. Actas Urol Esp. 2012; 36:449-60. [PubMed: 22386114]

7. David KA, Milowsky MI, Ritchey J, Carroll PR, Nanus DM. Low incidence of perioperative chemotherapy for stage III bladder cancer 1998 to 2003: a report from the National Cancer Data Base. J Urol. 2007; 178:451-4. [PubMed: 17561135]

8. Winquist E, Kirchner TS, Segal R, Chin J, Lukka H. Neoadjuvant chemotherapy for transitional cell carcinoma of the bladder: a systematic review and meta-analysis. J Urol. 2004; 171:561-9. [PubMed: 14713760]

9. Advanced Bladder Cancer Meta-analysis Collaboration. Neoadjuvant chemotherapy in invasive bladder cancer: a systematic review and meta-analysis. Lancet. 2003; 361:1927-34. [PubMed: 12801735]

10. Porter MP, Kerrigan MC, Donato BM, Ramsey SD. Patterns of use of systemic chemotherapy for Medicare beneficiaries with urothelial bladder cancer. Urol Oncol. 2011; 29:252-8. [PubMed: 19450992]

11. Leow, JJ.; Martin-Doyle, W.; Rajagopal, PS., et al. Adjuvant chemotherapy for invasive bladder cancer: a 2013 updated systematic review and meta-analysis of randomized trials. Eur Urol. In press. http://dx.doi.org/10.1016/j.eururo.2013.08.033

12. Bilimoria KY, Stewart AK, Winchester DP, Ko CY. The National Cancer Data Base: a powerful initiative to improve cancer care in the United States. Ann Surg Oncol. 2008; 15:683-90. [PubMed: 18183467]

13. Fedeli U, Fedewa SA, Ward EM. Treatment of muscle invasive bladder cancer: evidence from the National Cancer Database, 2003 to 2007. J Urol. 2011; 185:72-8. [PubMed: 21074192]

14. Rogers, EM. Diffusion of innovations. 5. New York, NY: Free Press; 2003.

15. Bamias A, Efstathiou E, Moulopoulos LA, et al. The outcome of elderly patients with advanced urothelial carcinoma after platinum-based combination chemotherapy. Ann Oncol. 2005; 16:30713. [PubMed: 15668289]

16. Hollenbeck BK, Miller DC, Taub D, et al. Aggressive treatment for bladder cancer is associated with improved overall survival among patients 80 years old or older. Urology. 2004; 64:292-7. [PubMed: 15302481]

17. von der Maase H, Sengelov L, Roberts JT, et al. Long-term survival results of a randomized trial comparing gemcitabine plus cisplatin, with methotrexate, vinblastine, doxorubicin, plus cisplatin in patients with bladder cancer. J Clin Oncol. 2005; 23:4602-8. [PubMed: 16034041]

18. Quan H, Li B, Couris CM, et al. Updating and validating the Charlson comorbidity index and score for risk adjustment in hospital discharge abstracts using data from 6 countries. Am J Epidemiol. 2011; 173:676-82. [PubMed: 21330339]

19. Boorjian SA, Kim SP, Tollefson MK, et al. Comparative performance of comorbidity indices for estimating perioperative and 5-year all cause mortality following radical cystectomy for bladder cancer. J Urol. 2013; 190:55-60. [PubMed: 23313198]

20. Gray PJ, Fedewa SA, Shipley WU, et al. Use of potentially curative therapies for muscle-invasive bladder cancer in the United States: results from the National Cancer Data Base. Eur Urol. 2013; 63:823-9. [PubMed: 23200811]

21. Thompson, RH.; Boorjian, SA.; Kim, SP., et al. Eligibility for neoadjuvant/adjuvant cisplatinbased chemotherapy among radical cystectomy patients. BJU Int. In press. http://dx.doi.org/ 10.1111/bju. 12274

22. Canter D, Viterbo R, Kutikov A, et al. Baseline renal function status limits patient eligibility to receive perioperative chemotherapy for invasive bladder cancer and is minimally affected by radical cystectomy. Urology. 2011; 77:160-5. [PubMed: 20709369]

23. Dash A, Galsky MD, Vickers AJ, et al. Impact of renal impairment on eligibility for adjuvant cisplatin-based chemotherapy in patients with urothelial carcinoma of the bladder. Cancer. 2006; 107:506-13. [PubMed: 16773629]

24. Mettlin CJ, Menck HR, Winchester DP, Murphy GP. A comparison of breast, colorectal, lung, and prostate cancers reported to the National Cancer Data Base and the Surveillance, Epidemiology, and End Results Program. Cancer. 1997; 79:2052-61. [PubMed: 9149035] 




Fig. 1.

Change in perioperative chemotherapy use between 2006 and 2010. Neoadjuvant chemotherapy increased from $10.1 \%$ in 2006 to $20.8 \%$ in $2010(p=0.005)$, whereas adjuvant chemotherapy remained stable between $18.1 \%$ and $21.3 \%(p=0.68)$. 
Table 1

Analysis of patient characteristics

\begin{tabular}{|c|c|c|c|c|}
\hline & $\mathrm{RC}$ only, $n(\%)$ & NAC and RC, $n(\%)$ & $\mathrm{RC}$ and $\mathrm{AC}, n(\%)$ & $p$ value \\
\hline \multicolumn{5}{|l|}{ Age, yr } \\
\hline$<50$ & $195(5.4)$ & $100(10.4)$ & $87(7.9)$ & $<0.001$ \\
\hline $50-59$ & $590(16.2)$ & $255(26.5)$ & $262(23.9)$ & \\
\hline $60-69$ & $1058(29.1)$ & $335(34.8)$ & $398(36.4)$ & \\
\hline $70-79$ & $1219(33.5)$ & $229(23.8)$ & $296(27.1)$ & \\
\hline 280 & $574(15.8)$ & $43(4.5)$ & $51(4.7)$ & \\
\hline Gender & & & & 0.59 \\
\hline Male & $2455(67.5)$ & $666(69.2)$ & $740(67.6)$ & \\
\hline Female & $1181(32.5)$ & $296(30.1)$ & $354(32.4)$ & \\
\hline Race & & & & 0.81 \\
\hline White & $3301(90.8)$ & $883(91.8)$ & $993(90.8)$ & \\
\hline Black & $210(5.8)$ & $45(4.7)$ & $66(6.0)$ & \\
\hline Other & $88(2.4)$ & $23(2.4)$ & $22(2.0)$ & \\
\hline Unknown & $37(1.0)$ & $11(1.1)$ & $13(1.2)$ & \\
\hline Charlson Comorbidity Index sum & & & & $<0.001$ \\
\hline 0 & $2461(67.7)$ & $747(77.7)$ & $785(71.8)$ & \\
\hline 1 & $888(24.4)$ & $172(18.0)$ & $251(22.9)$ & \\
\hline$>1$ & $287(7.9)$ & $42(4.4)$ & $58(5.3)$ & \\
\hline Insurance status & & & & $<0.001$ \\
\hline Not insured & $136(3.7)$ & $34(3.5)$ & $36(3.3)$ & \\
\hline Private/managed care & $1120(30.8)$ & $438(45.5)$ & $463(42.3)$ & \\
\hline Medicaid & $166(4.6)$ & $53(5.5)$ & $55(5.0)$ & \\
\hline Medicare & $2141(58.9)$ & $413(42.9)$ & $516(47.2)$ & \\
\hline VA/military & $29(0.8)$ & $6(0.6)$ & $9(0.8)$ & \\
\hline Unknown & $44(1.2)$ & $18(1.9)$ & $15(1.4)$ & \\
\hline Geographic location & & & & $<0.001$ \\
\hline Northeast & $700(19.3)$ & $242(25.2)$ & $260(23.8)$ & \\
\hline South/southeast & $981(27.0)$ & $254(26.4)$ & $262(24.0)$ & \\
\hline Midwest & $1096(30.1)$ & $299(31.1)$ & $346(31.6)$ & \\
\hline West & $859(23.6)$ & $167(17.4)$ & $226(20.7)$ & \\
\hline Facility type & & & & $<0.001$ \\
\hline Community center & $392(10.8)$ & $89(9.3)$ & $158(14.4)$ & \\
\hline Comprehensive community cancer center & $1652(45.4)$ & $313(32.5)$ & $495(45.2)$ & \\
\hline Academic/Research center & $1561(42.9)$ & $558(58.0)$ & 434 (39.7) & \\
\hline Other & $31(0.9)$ & $2(0.2)$ & $7(0.6)$ & \\
\hline Distance from hospital & & & & $<0.001$ \\
\hline Same ZIP code & $228(6.3)$ & $56(5.8)$ & $97(8.9)$ & \\
\hline
\end{tabular}

Eur Urol. Author manuscript; available in PMC 2015 July 01. 


\begin{tabular}{|c|c|c|c|c|}
\hline & RC only, $n(\%)$ & $\mathrm{NAC}$ and $\mathrm{RC}, n(\%)$ & $\mathrm{RC}$ and $\mathrm{AC}, n(\%)$ & $p$ value \\
\hline$\$ 60$ miles & $2588(71.2)$ & $655(68.1)$ & $841(76.9)$ & \\
\hline $61-120$ miles & $368(10.1)$ & $110(11.4)$ & $67(6.1)$ & \\
\hline$>120$ miles & $452(12.4)$ & $141(14.7)$ & $89(8.1)$ & \\
\hline Rurality & & & & 0.78 \\
\hline Metro & $2640(77.7)$ & $700(78.3)$ & $827(79.5)$ & \\
\hline Urban & $659(19.4)$ & $167(18.7)$ & $187(18.0)$ & \\
\hline Rural & $91(2.9)$ & $27(3.0)$ & $26(2.5)$ & \\
\hline Income & & & & 0.002 \\
\hline$<\$ 30000$ & $445(13.1)$ & $81(8.9)$ & $119(11.4)$ & \\
\hline$\$ 30000-34999$ & 715 (21.) & $164(18.1)$ & $199(19.1)$ & \\
\hline$\$ 35000-45999$ & $997(29.3)$ & $283(31.2)$ & $324(31.0)$ & \\
\hline$\$ \$ 46000$ & $1249(36.7)$ & $378(41.7)$ & $402(38.5)$ & \\
\hline
\end{tabular}

$\mathrm{AC}=$ adjuvant chemotherapy $\mathrm{NAC}=$ neoadjuvant chemotherapy $; \mathrm{RC}=$ radical cystectomy $; \mathrm{VA}=$ Veterans Affairs. 
Table 2

Multivariable analysis of covariates associated with delivery of perioperative chemotherapy

\begin{tabular}{|c|c|c|c|}
\hline Factor & Odds ratio & $95 \% \mathrm{CI}$ & $p$ value \\
\hline Year of diagnosis & 1.11 & $1.07-1.17$ & $<0.01$ \\
\hline \multicolumn{4}{|l|}{ Age, yr } \\
\hline$<50$ & Referent & & \\
\hline $50-59$ & 0.89 & $0.69-1.15$ & 0.37 \\
\hline $60-69$ & 0.73 & $0.57-0.94$ & 0.02 \\
\hline $70-70$ & 0.45 & $0.33-0.59$ & $<0.01$ \\
\hline 280 & 0.16 & $0.11-0.23$ & $<0.01$ \\
\hline \multicolumn{4}{|l|}{ Race } \\
\hline White & Referent & & \\
\hline Black & 0.85 & $0.65-1.10$ & 0.22 \\
\hline Other & 0.83 & $0.56-1.25$ & 0.38 \\
\hline Unknown & 1.03 & $0.58-1.83$ & 0.92 \\
\hline \multicolumn{4}{|l|}{ Gender } \\
\hline Male & Referent & & \\
\hline Female & 1.10 & $0.97-1.25$ & 0.15 \\
\hline \multicolumn{4}{|c|}{ Charlson Comorbidity Index sum } \\
\hline 0 & Referent & & \\
\hline 1 & 0.86 & $0.74-0.99$ & 0.04 \\
\hline$>1$ & 0.64 & $0.49-0.82$ & $<0.01$ \\
\hline \multicolumn{4}{|l|}{ Insurance status } \\
\hline Private/managed care & Referent & & \\
\hline Uninsured & 0.64 & $0.46-0.89$ & $<0.01$ \\
\hline Medicaid & 0.80 & $0.60-1.06$ & 0.12 \\
\hline Medicare & 0.94 & $0.8-1.10$ & 0.44 \\
\hline VA/military & 0.97 & $0.49-1.91$ & 0.93 \\
\hline Unknown & 0.79 & $0.46-1.36$ & 0.40 \\
\hline \multicolumn{4}{|l|}{ Geographic location } \\
\hline Northeast & Referent & & \\
\hline South & 0.70 & $0.59-0.85$ & $<0.01$ \\
\hline Midwest & 0.79 & $0.67-0.94$ & $<0.01$ \\
\hline West & 0.64 & $0.54-0.79$ & $<0.01$ \\
\hline \multicolumn{4}{|l|}{ Distance from hospital } \\
\hline Same ZIP code & Referent & & \\
\hline$<60$ miles & 0.81 & $0.63-1.02$ & 0.08 \\
\hline $60-120$ miles & 0.64 & $0.47-0.89$ & $<0.01$ \\
\hline$>120$ miles & 0.64 & $0.46-0.89$ & 0.01 \\
\hline Rurality & & & \\
\hline
\end{tabular}




\begin{tabular}{clll}
\hline Factor & Odds ratio & $\mathbf{9 5 \%}$ CI & $\boldsymbol{p}$ value \\
\hline Metro & Referent & & \\
\hline Urban & 1.03 & $0.87-1.23$ & 0.70 \\
\hline Rural & 1.17 & $0.80-1.71$ & 0.42 \\
\hline
\end{tabular}

\begin{tabular}{llll}
\hline Hospital type & & & \\
\hline Community cancer program & Referent & & \\
\hline Comprehensive community cancer program & 0.86 & $0.70-1.06$ & 0.17 \\
\hline Academic & 1.08 & $0.87-1.33$ & 0.49 \\
\hline Other & 0.72 & $0.33-1.59$ & 0.42 \\
\hline Median annual income & & & \\
\hline$<\$ 30000$ & Referent & & \\
\hline$\$ 30000-34999$ & 1.11 & $0.89-1.39$ & 0.36 \\
\hline$\$ 35000-45999$ & 1.26 & $1.01-1.56$ & 0.04 \\
\hline $2 \$ 46000$ & 1.28 & $1.03-1.59$ & 0.03 \\
\hline
\end{tabular}

$\mathrm{CI}=$ confidence interval; $\mathrm{VA}=$ Veterans Affairs. 